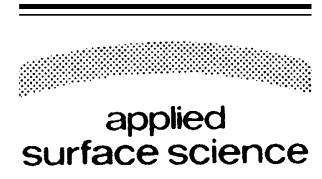

Applied Surface Science 231-232 (2004) 59-63

www.elsevier.com/locate/apsusc

\title{
Energetic cluster induced desorption from a graphite surface
}

\author{
R. Webb* \\ Advanced Technology Institute, University of Surrey, Guildford GU2 7XH, Surrey, UK
}

Available online 30 April 2004

\begin{abstract}
Using molecular dynamics computer simulation it has been shown how a large cluster such as fullerene can create an acoustic wave with sufficient energy to gently eject molecules which have been adsorbed to a surface. In a preliminary study we demonstrated how this acoustic wave can be engineered to increase in power by using a larger cluster. It is shown that ejection/ desorption of adsorbate molecules via this technique can result in extremely high $(\sim 100 \%)$ fractions of intact particles and result in little or no breakage of remaining molecules or the host material provided the impact energy is kept sufficiently low. Analysis of our simulation results shows that the molecular yield increases logarithmically with energy and highlights the effectiveness of this desorption process. We also demonstrate the desorption of a large intact cluster $\mathrm{C}_{300}$ adsorbed on the surface via a collision of a smaller cluster $\mathrm{C}_{60}$.
\end{abstract}

(C) 2004 Elsevier B.V. All rights reserved.

Keywords: Cluster impact desorption; Computer simulation; Molecular dynamics

\section{Introduction}

The ejection of large intact molecules from a surface using employing an ion beam is of much interest to the mass spectrometry community and of particular interest to workers using SIMS to image biological systems. One recent development on this has been the development of poly-atomic ion sources, in particular, a fullerene source [1]. As space is limited here no discussion will be developed on this. Previously we have demonstrated [2] using molecular dynamics computer simulation that the energetic interaction of $\mathrm{C}_{60}$ fullerene with a graphite surface can produce an acoustic wave, which can result in the ejection of small molecules (benzene) which had previously been adsorbed to the surface [3-5]. It was also shown $[3,4]$

\footnotetext{
*Tel.: +44-1483-689830; fax: +44-1483-686091. E-mail address: r.webb@ee.surrey.ac.uk (R. Webb).
}

that if the energy of the striking molecule was kept low enough $(<400 \mathrm{eV})$ then the impact, whilst sufficient to cause ejection of intact adsorbed molecules, was not enough to cause breakage of the molecules even when hit directly by the incoming fullerene. The problem with such low energy impacts is that the number of ejected molecules is relatively small, only of the order of 10-20 benzene molecules are ejected from a single $\mathrm{C}_{60}$ collision. If the energy of the $\mathrm{C}_{60}$ is increased to obtain a greater yield then it starts to break-up the adsorbed surface layer of the benzene molecules and creates substantial debris on the surface from the benzene break-up, the surface damage and the destruction of the impacting fullerene. As a method of increasing the energy put into the acoustic wave responsible for ejecting the intact molecules the work reported previously attempted using larger fullerene structures, such as a double skinned $\mathrm{C}_{300}$ and a triple skinned $\mathrm{C}_{840}$. This work demonstrated that yields as 
high as 70 intact benzene molecules per incident cluster can be achieved without causing any surface degradation. The purpose of the work reported here is to explore further this phenomenon and explore this mechanism in the ejection of large molecules adsorbed on graphite, employing higher velocity clusters.

\section{Simulation method}

The molecular dynamics model used for this work has been described in detail elsewhere [6,7], consequently, only a short description will be given here. The Brenner many body interaction potential [8] is used to describe the interactions between the $\mathrm{C}-\mathrm{C}$ and $\mathrm{C}-\mathrm{H}$ atoms in the simulation. This potential has been found to provide a good description of the $\mathrm{C}-\mathrm{H}$ system under the energetic conditions employed here and produces stable molecular configurations for the hydro-carbons simulated. However the cut-off distance of this potential is such that there is no interplaner bonding between graphite layers and no Van der Waals attraction forces between molecules close to a graphitic surface. To overcome this a long range term is added, based on the Lennard-Jones pair-wise potential [9]. This additional interaction potential has a cutoff point which is dependent on the number of bonds connecting the two atoms under consideration to ensure that the long-range term does not add to intra-layer bonding. A variety of target types have been used in the results presented here but in general the simulations have been performed for a small number (29) of different impact points and mainly on a graphite target with an over-layer of benzene molecules adsorbed on the surface. The molecules in the adsorbate layer are set above the initial graphite surface so that they are confined within the potential minimum created by the long-range potential and are effectively bond to the surface by a weak Van der Waals force $(\sim 0.5 \mathrm{eV}$ per molecule). The targets used are typically $100 \AA \times 100 \AA \times 40 \AA$ thick, although thinner targets have been used in some situations to save on computation time. The target is constructed with all atoms in their equilibrium positions with no initial kinetic energy. We use free boundaries to avoid reflection of energy back from the edges of the crystallite. In the simulations, looking at the deso- rption as a function of impact angle periodic boundaries were used as for grazing incidence the flight path of the cluster can be quite long compared to the size of the crystallite. With any simulation of this type the weak part is always the behaviour of the crystallite boundaries and there is no guaranteed method of providing the correct behaviour. Consequently, the best situation is to keep the boundaries as far away as possible from the observation region of the simulation, which is what we have attempted to do in the results presented here.

\section{Results and discussion}

Presented in Fig. 1 is the total molecular yield of benzene (or cumene) ejected from impacts on overlayers on graphite and diamond substrates as a function of energy. The dotted lines are logarithmic curves fitted to the higher energy data points. For the higher energy impacts $(\sim 1-8 \mathrm{keV})$ the molecular yield increases approximately logarithmically with energy. At lower energy $(<1 \mathrm{keV})$ the yield tends to be higher for the graphite substrate, but consistent for the diamond substrate. The reason for this has been explained elsewhere [4] and is due to the production of the acoustic wave in the surface and the sticking or reflection of the cluster after impact.

In Fig. 2 we show how the position of ejection from the impact site changes with energy. As can be seen there is a distinct difference between the behaviour of the diamond substrate compared with the graphite substrate. In the case of the graphite substrate the distance from the impact site increases sharply with energy - as the acoustic wave increase in strengthand then flattens out to a constant. Whereas for the diamond target the distance increases quickly initially but then rises more slowly with energy to saturate at about the same value. The initial fast rise is thought to be due to the direct interaction of the cluster with the adsorbate over-layer, which peaks at around $300 \mathrm{eV}$, which is similar for both diamond and graphite. The higher yield and faster saturation of distance from impact in the case of graphite is caused by the easier production of the surface acoustic wave. This is to some extent borne out by the change in the velocity distributions shown in Fig. 3 for a range of energies for the graphite substrate with a benzene over-layer. The 


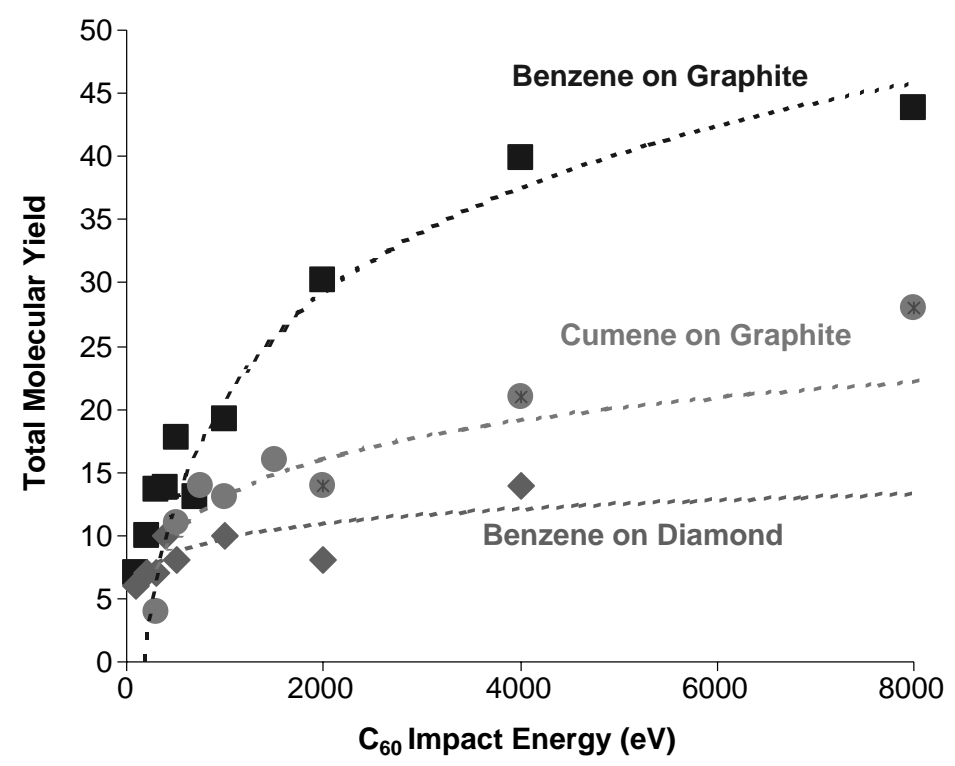

Fig. 1. Total molecular ejection yield as a function of cluster impact energy from an adsorbed molecular over-layer of benzene and cumene on graphite and diamond substrates.

distributions show quite a broad shape at very low energy $(300 \mathrm{eV})$ when the cluster interacts strongly with the over-layer itself and then becomes narrower up to about $1 \mathrm{keV}$ impact energy when the cluster penetrates the graphite and tends to stick in the surface region dampening the acoustic waves produced, giving rise to relatively low energy (velocity) ejection. At higher energy still $(>1 \mathrm{keV})$ the cluster penetrates the

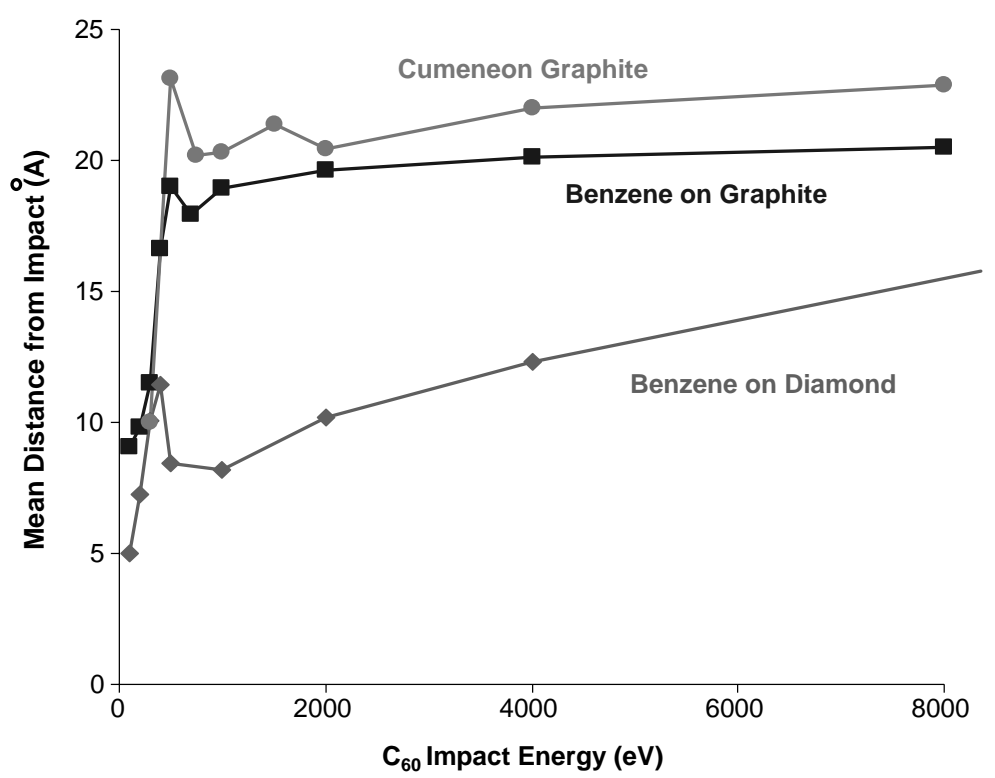

Fig. 2. The mean distance of an ejected molecule from the impact site of a cluster as a function of energy for an adsorbed molecular over-layer of benzene and cumene on graphite and diamond substrates. 


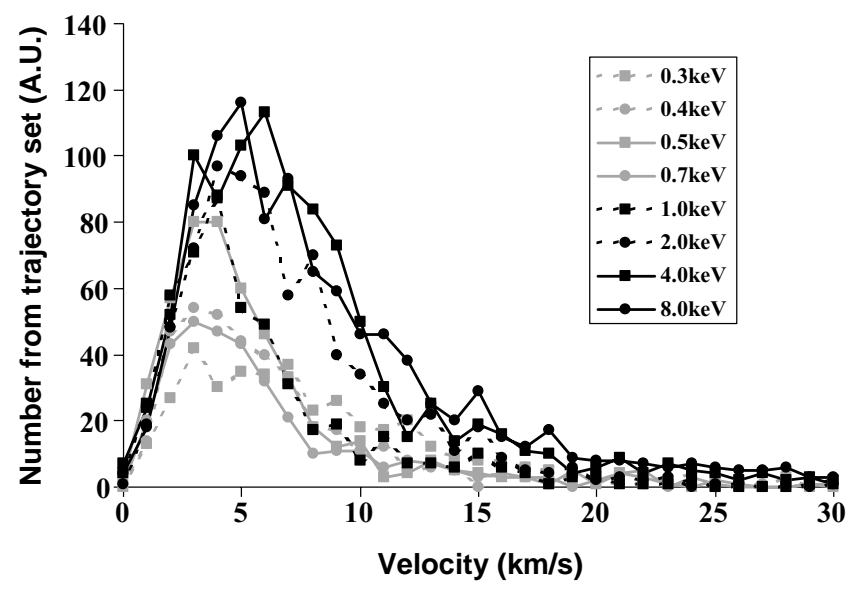

Fig. 3. Velocity distributions of ejected benzene molecules from a set of $\mathrm{C}_{60}$ cluster impacts on an adsorbed benzene over-layer on a graphite substrate.

graphite deeply enough, allowing the acoustic wave to assist in the ejection process again.

As mentioned in Section 1 previous simulations have been performed using larger clusters to enhance the yield of intact molecules at lower impact energies. Comparing the yields from a $\mathrm{C}_{60}$ cluster impact to that of a $\mathrm{C}_{300}$ and a $\mathrm{C}_{840}$ cluster impacts at low energies maintaining the same velocity of the cluster then the yields, normalised to the number of atoms in the cluster, become comparable for the $\mathrm{C}_{300}$ case but are reduced for the $\mathrm{C}_{840}$ case. It is not clear at this time if the reduction in yield from the $\mathrm{C}_{840}$ is because the simulations have not been run for long enough times or if this is a genuine effect. There is some

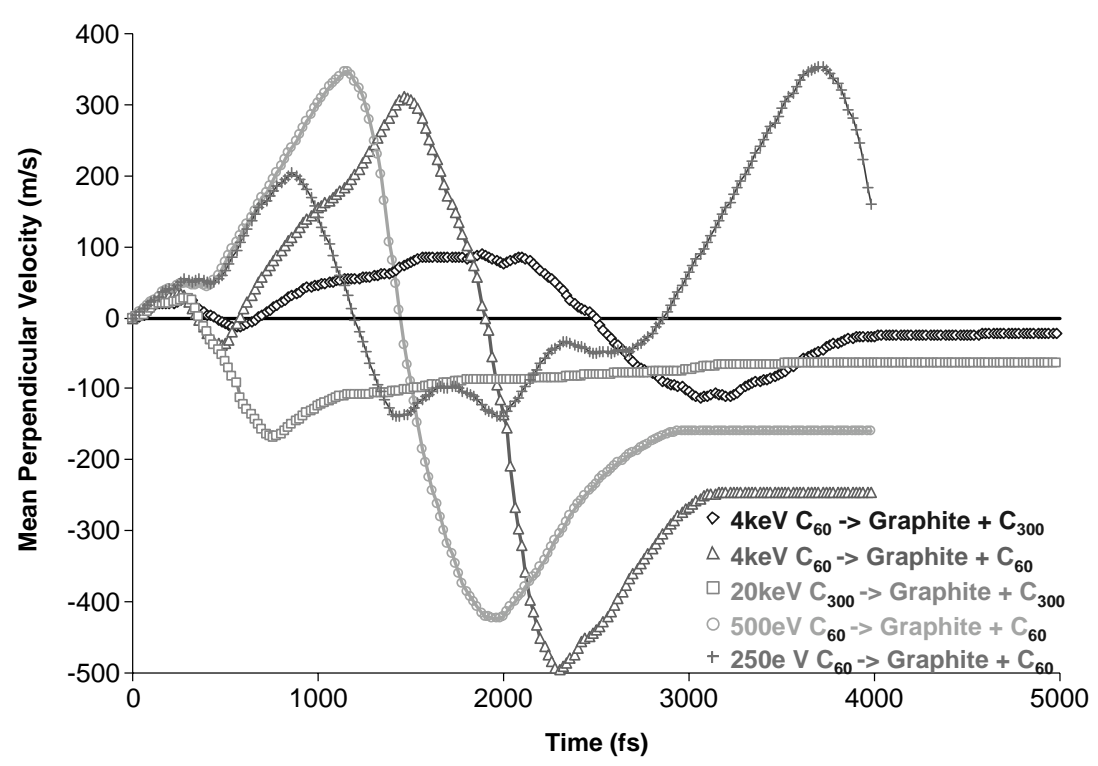

Fig. 4. The mean perpendicular velocity of different molecules adsorbed to a graphite substrate and struck by an energetic cluster as a function of time. A positive velocity is in the direction of the surface and a negative velocity is away from the surface. In all cases the adsorbed molecule is ejected except for the lowest energy impact, which show the molecule to be still attached to the surface. 
evidence that the $\mathrm{C}_{840}$ impacts have not completed after 4 ps whereas the $\mathrm{C}_{300}$ and $\mathrm{C}_{60}$ impacts are much further on. The difference in timing comes about because the larger clusters cause more layers in the substrate to be included in the acoustic wave, which slows its progress.

The question that still remains, however, is: can this acoustic wave cause the ejection of a large intact molecule from the surface of a graphite target? To answer this we have adsorbed a large molecule to the surface using Van der Waals forces. The molecules we have chosen is $\mathrm{C}_{60}$ and $\mathrm{C}_{300}$. We have then struck the surface close-by ( $1 \mathrm{~nm}$ away) by a $\mathrm{C}_{60}$ or a $\mathrm{C}_{300}$ and watched the perpendicular velocity of the adsorbed molecule on the surface to see if it leaves the target. The results are shown in Fig. 4. A positive velocity means the molecule is moving towards the surface and a negative velocity indicates movement away from the surface. We see that if the initial energy of the cluster is high enough we can create an acoustic wave that is strong enough to cause the adsorbed fullerene to be ejected. A $4 \mathrm{keV} \mathrm{C}_{60}$ molecule can create a surface wave large enough to knock off an adsorbed $\mathrm{C}_{300}$ molecule $1 \mathrm{~nm}$ away from the impact site. However, it should be noted that at $250 \mathrm{eV}$ the $\mathrm{C}_{60}$ impact failed to eject an adsorbed $\mathrm{C}_{60}$ molecule, it remained bound to the surface despite being "shaken".

\section{References}

[1] D. Weibel, S. Wong, N. Lockyer, P. Blenkinsopp, R. Hill, J. Vickerman, Anal. Chem. (accepted for publication).

[2] R. Smith, R. Webb, Proc. R. Soc. London A 441 (1993) 495.

[3] R. Webb, M. Kerford, E. Ali, M. Dunn, L. Knowles, K. Lee, J. Mistry, F. Whitefoot, Surf. Interface Anal. 31 (2001) 297.

[4] M. Kerford, R. Webb, Nucl. Instrum. Methods B 180 (2001) 44.

[5] M. Kerford, R. Webb, Nucl. Instrum. Methods B 153 (1999) 270.

[6] D.E. Harrison Jr., Crit. Rev. Solid State Mater. Sci. 14 (1989) S1.

[7] R. Smith, D.E. Harrison Jr., B.J. Garrison, Phys. Rev. B 40 (1989) 93.

[8] D.W. Brenner, Phys. Rev. B 46 (1992) 1948.

[9] R. Smith, K. Beardmore, Thin Solid Films 272 (1996) 255. 